\title{
Curso clínico de mulheres grávidas com infecção pelo SARS-CoV-2 com desenvolvimento da COVID-19: revisão integrativa da literatura
}

Clinical course of pregnant women with SARS-CoV-2 infection with development of COVID-19: an integrative literature review

Evolución clínica de mujeres embarazadas con infección por SARS-CoV-2 con desarrollo de COVID-19: una revisión bibliográfica integradora

\begin{abstract}
Resumo
A pandemia de SARS-CoV-2 acarreta impactos à saúde ainda desconhecidos e que são objetivos de estudos. Dentre esses, estão a forma como a COVID-19 se manifesta em gestantes e puérperas, quais os desfechos neonatais e qual o perfil epidemiológico dessas mulheres. Para tanto foi conduzida uma Revisão integrativa da literatura nas bases de dados PubMed. Os descritores utilizados para delimitar as buscas foram gravidez, SARS-CoV-2, COVID-19. Assim sendo, 27 artigos foram selecionados ao final das buscas. Sendo 92,6\% deles publicados ano de 2020, com relação ao país de origem de realização dos estudos o mais prevalente com 8 (29,62\%) artigos, foram os Estados Unidos. Evidências apontam que há relações entre maior necessidade de internação em Unidade de Terapia Intensiva e uso de suporte ventilatório entre gestantes com COVID-19. A gestação parece estar relacionada com formas severas da doença, porém, existem outros fatores influenciadores, como obesidade e diabetes mellitus. Houve maior proporção de gestantes negras e latinas entre as afetadas, com aparente aumento no risco de morte entre as latinas. Também, notou-se um aumento nos partos pré-termo quando da forma severa. Dessa forma, fica evidente que há uma relação entre a chance de agravamento da COVID-19 entre gestantes com SARS-CoV-2, em especial as sintomáticas e com comorbidades. Além disso, aumento da taxa de partos pré-termo com a forma severa. Assim, adotar medidas de prevenção e vacinação entre essa população se mostra como as principais formas de evitar agravamentos, partos prétermo e óbitos.
\end{abstract}

Palavras-chave: Gravidez; SARS-CoV-2; COVID-19.

\begin{abstract}
The SARS-CoV-2 pandemic causes health impacts that are still unknown and that are objectives of studies. Among these are how COVID-19 manifests itself in pregnant and postpartum women, what are the neonatal outcomes and what is the epidemiological profile of these women. To this end, an integrative literature review was conducted in the PubMed databases. The descriptors used to delimit the searches were pregnancy, SARS-CoV-2, COVID-19. Thus, 27 articles were selected at the end of the search. $92.6 \%$ of them were published in 2020, and the most prevalent country of origin was the United States, with 8 (29.62\%) articles. Evidence shows that there is a relationship between a greater need for admission to the Intensive Care Unit and the use of ventilatory support among pregnant women with COVID-19. Pregnancy seems to be related to severe forms of the disease, but there are other influencing factors, such as obesity and diabetes mellitus. There was a higher proportion of black and Latina pregnant women among those
\end{abstract}


affected, with an apparent increase in the risk of death among Latinas. Also, an increase in preterm births was noted when the severe form was present. Thus, it is evident that there is a relationship between the chance of worsening COVID-19 among pregnant women with SARS-CoV-2, especially those who are symptomatic and have comorbidities. In addition, increased rate of preterm births with the severe form. Thus, adopting preventive measures and vaccination among this population show as the main ways to avoid worsening, preterm births and deaths.

Keywords: Pregnancy; SARS-CoV-2, COVID-19.

\begin{abstract}
Resumen
La pandemia de SARS-CoV-2 conlleva impactos sanitarios que aún se desconocen y que son objetivo de estudios. Entre ellos, están la forma en que la COVID-19 se manifiesta en gestantes y puérperas, cuáles son los desfases neonatales y cuál es el perfil epidemiológico de las mujeres. Para ello, se realizó una revisión bibliográfica integradora en las bases de datos de PubMed. Los descriptores utilizados para delimitar las búsquedas fueron embarazo, SARSCoV-2, COVID-19. Por lo tanto, al final de la búsqueda se seleccionaron 27 artículos. El 92,6\% de ellos se publicaron en 2020, y el país de origen más frecuente fue Estados Unidos, con 8 (29,62\%) artículos. La evidencia muestra que existe una relación entre la mayor necesidad de ingreso en la Unidad de Cuidados Intensivos y el uso de soporte ventilatorio entre las mujeres embarazadas con COVID-19. El embarazo parece estar relacionado con las formas graves de la enfermedad, pero hay otros factores que influyen, como la obesidad y la diabetes mellitus. Había una mayor proporción de mujeres embarazadas negras y latinas entre las afectadas, con un aparente aumento del riesgo de muerte entre las latinas. También se observó un aumento de los nacimientos prematuros en la forma grave. Por lo tanto, es evidente que existe una relación entre la probabilidad de empeoramiento del COVID-19 entre las mujeres embarazadas con SARS-CoV-2, especialmente las sintomáticas y las que presentan comorbilidades. Además, un aumento de la tasa de nacimientos prematuros con la forma grave. Así, la adopción de medidas preventivas y la vacunación entre esta población son las principales vías para evitar el empeoramiento, los partos prematuros y las muertes.
\end{abstract}

Palabras clave: Embarazo; SARS-CoV-2; COVID-19.

\title{
1. Introdução
}

A doença Coronavírus 2019 (COVID-19) tem como etiologia um betacoronavírus de RNA do subgênero Sarbecovirus nomeado de SARS-CoV-2 (Severe acute respiratory syndrome coronavirus-2), cujo receptor é o da Enzima Conversora de Angiotensina 2 (ECA2), presente no epitélio alveolar dos pulmões, enterócitos, endotélio e células musculares de artérias e endotélio venoso. As proteínas "spike" do vírus ligam-se nos receptores dando início ao processo infeccioso e replicação viral (Lim et al., 2020; Wenling et al., 2020). O SARS-CoV-2 é altamente contagioso, transmitido majoritariamente por via de gotículas respiratórias, ocasionando desde casos assintomáticos até de Síndrome Respiratória Aguda Grave (SRAG) e acometimento sistêmico (Wenling et al., 2020).

Ter conhecimento sobre os riscos em gestantes se torna importante, visto que as complicações da doença podem influenciar e trazer consequências não apenas para mãe, mas também para o feto, principalmente durante o terceiro trimestre de gestação, como é relatado em estudos de Rajewska et al. (2020), que evidenciou parto prematuro, descolamento prévio de placenta e sofrimento fetal.

De acordo com Wu et al (2020), as manifestações clinicas decorrentes da infecção pelo vírus SARS-CoV-2 variam de pacientes assintomáticos, ou seja, possuíam o vírus, porém, não manifestavam quaisquer sintomas a quadros gripais. Os sintomas gripais tornaram-se as manifestações mais comuns, tais como: dispneia, lacrimejamento, coriza hialina, febre, tosse, mialgia e fadiga. Além disso, outros sintomas clínicos também são comumente encontrados, sendo eles: diarreia, cefaleia e lombalgia. Em alguns casos mais graves, o acometimento pulmonar pode gerar quadros graves respiratórios, levando a um desequilíbrio das atividades corporais e, em inúmeros casos, queda brusca da saturação de oxigênio, gerando hipóxia severa.

Quando comparado as manifestações em mulheres, percebeu-se que, as gestantes são particularmente mais vulneráveis a patógenos respiratórios e pneumonia grave devido a alterações fisiológicas: aumento do consumo de oxigênio, diminuição da capacidade residual funcional e diminuição complacência torácica, e imunológicas: relacionadas às alterações nos perfis de ação dos linfócitos T, principalmente os $\mathrm{T}$ helper, desta forma resultando em maior morbidade e mortalidade fetal 
(Wenling et al, 2020). Além disso, alguns fatores de risco acarretam em maior complexidade durante o período gestacional, como, por exemplo, grávidas que apresentam pneumonia, têm um risco maior de dar à luz a um recém-nascido com baixo peso, bem como prematuros e, pequeno para a idade gestacional.

Baseado no exposto, o objetivo desse estudo foi mostrar, analisar e reportar as manifestações clínicas e complicações em gestantes afetadas pelo SARS-CoV-2, bem como os principais desfechos pós infecção.

\section{Metodologia}

Neste estudo foi realizada uma revisão integrativa, ou seja, uma pesquisa para avaliar, sintetizar e conhecer a sobre um fenômeno a partir de evidências, com objetivo de proporcionar um panorama de teorias, conceitos complexos e questões de saúde pertinentes por meio de estudos já realizados, proporcionando a apresentação intervenção (Galvão et al., 2004; Whittemore \& Knafl, 2005).

Para a separação dos artigos, foram realizadas 6 partes metodológicas, são elas: 1. foi detectado o problema, aplicado o mecanismo de busca e os descritores para se formular a questão guiadora ou hipótese; 2. estabelecido os critérios de inclusão e exclusão dos artigos a serem selecionados para integrar a amostra; 3. leitura e resumo dos artigos para a seleção; 4. informações dos artigos foram compiladas, analisadas e agrupadas; 5. resultados foram detectados. 6. resumo após a exposição dos resultados verificados, que intermeiam a hipótese da pesquisa (De Sousa et al., 2011).

Desse modo, destinou-se os seguintes conceitos para a realização da busca: gravidez, SARS-CoV-2, COVID-19, a pergunta norteadora, baseada em tais conceitos, é: Qual o papel da infecção pelo SARS-CoV-2, no desenvolvimento da COVID-19 em mulheres gestantes e no desfecho da gestação?

Após definir a questão a ser pesquisada, foi realizado uma pesquisa e seleção bibliográfica na plataforma PubMed. A seleção do estudo ocorreu entre os anos de 2019 e 2021. E a escolha dos textos procedeu com as buscas na plataforma, utilizando os filtros nela disponível para textos publicados entre fevereiro e março de 2021. Para seleção das publicações, foram adotados os seguintes critérios de inclusão: artigos científicos, publicados no idioma Inglês, entre os anos de 2019 a 2021, disponíveis online e gratuitamente na íntegra. Foram excluídos os artigos sem resumo na base de dados ou incompletos, editoriais, cartas ao editor, estudos reflexivos, revisões sistemáticas ou integrativas de literatura.

Após a definição do assunto chave para este artigo de revisão e da seleção dos artigos a serem analisados, foram identificadas 2712 publicações com grandes chances de elegibilidade para serem incluídas nessa revisão. Por conseguinte, após a aplicação dos critérios de inclusão e exclusão, a amostra foi composta por 395 publicações. Foi feita a análise dos resumos destes 37 registros, para verificar se atenderiam os critérios de elegibilidade e se responderiam à pergunta que norteia este artigo de revisão. Sendo assim, foram excluídos 10 registros e apenas 27 foram analisados na íntegra para a síntese e análise dos dados conforme o fluxograma de seleção (Figura 1). 
Research, Society and Development, v. 10, n. 13, e168101321083, 2021

(CC BY 4.0) | ISSN 2525-3409 | DOI: http://dx.doi.org/10.33448/rsd-v10i13.21083

Figura 1: Representação da estratégia de seleção dos artigos científicos na base de dados.

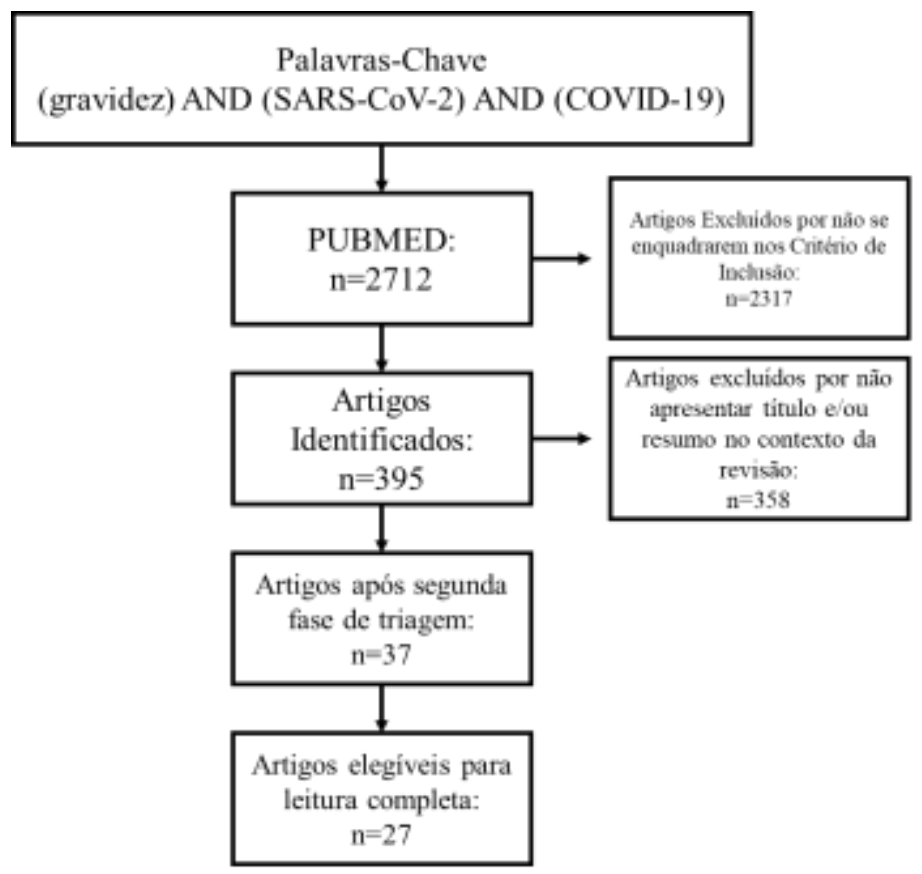

Fonte: Autores (2021).

\section{Resultados e Discussão}

No espaço de tempo delimitado para a realização deste estudo (2019-2021) foram encontradas e analisadas 27 publicações, sendo que 92,6\% foram publicados no ano de 2020 e 7,4\% foram publicados em 2021.

De acordo com a metodologia dos trabalhos selecionados, os tipos de estudos eram pesquisas que analisaram a relação entre COVID - 19 e suas consequências na gestação em diversos centros médicos do mundo.

As publicações resultaram de diferentes revistas sendo: The BMJ, Turkish Journal of Obstetrics and Gynecology, The Journal of Maternal-Fetal \& Neonatal Medicine, Wiley Online Library, American Journal of obstetrics \& Gynecology, European Journal of Pediatrics, Royal College of Obstetricians and Gynaecologists, Morbidity and Mortality Weekly Report (MMWR), Morbidity and Mortality Weekly Report, American Journal of Obstetrics \& Gynecology MFM, BMC Pregnancy and Childbirth, Actas Obstetricia et Gynecologica Scandinavica, Elsevier, Acta Paediatrica, Clinical Infectious Diseases, The Lancet, International Journal of Infectious Diseases, Ultrasound in Obstetrics e Ginecology,

Analisando os locais de estudo, 8 artigos foram realizados nos Estados Unidos (29,62\%), 2 artigos no Reino Unido (7,4\%), 2 artigos na Turquia (7,4\%), 2 artigos na Itália (7,4\%), 3 artigos realizados no Brasil (11,11\%), 2 artigos na Espanha (7,4\%), 4 artigos na China (14,81\%) e em cada um desses países, sendo eles, México (3,7\%), Kuwait (3,7\%), Suécia (3,7\%) e Irã $(3,7 \%)$ foram publicados um artigo respectivamente.

A Tabela 1, sumariza os estudos selecionados nos seguintes aspectos, quais sejam: grupo étnico; necessidade de internação; idade gestacional no momento da internação; gravidade dos sintomas; necessidade de internação em UTI; utilização de ventilação mecânica; mortalidade materna e mortalidade fetal.

Estudo conduzido por Ellington e Cols (2020), tendo como objetivo analisar os dados de 91.412 relatórios médicos disponibilizados pelo CDC nos Estados Unidos com teste laboratorial confirmado para SARS-CoV-2 e status de gravidez a fim de determinar a relação de morbidade e mortalidade em mulheres grávidas e não grávidas. Dentre os resultados, foi evidenciado que mulheres grávidas e não grávidas tiveram frequência similar de tosse e dispneia, no entanto, grávidas 
Research, Society and Development, v. 10, n. 13, e168101321083, 2021

(CC BY 4.0) | ISSN 2525-3409 | DOI: http://dx.doi.org/10.33448/rsd-v10i13.21083

apresentaram com menos frequência dor de cabeça, mialgia, febre, calafrios e diarreia. Gestantes apresentaram taxas de internação hospitalar significativamente maiores (não foi possível distinguir internação por razões obstétricas de internação por COVID-19), totalizando 5.4 vezes mais chance de hospitalização. Além disso, o estudo mostra que gestantes são mais susceptíveis à internação em UTI (1.5 maior) e a necessitar de ventilação mecânica (1.7 vezes maior). Não houve diferença no risco de morte e entre as grávidas em internação hospitalar, internação em UTI e uso de ventilação mecânica a faixa etária mais frequente foi entre 35 e 44 anos. Os dados disponíveis sugerem que há maior proporção de mulheres hispânicas e negras grávidas afetadas pela COVID-19 durante a gestação, no entanto não estão disponíveis os dados étnicos de $20 \%$ das gestantes. Em estudo conduzido por Lokken e Cols (2020), também observaram mior incidência em pacientes gestantes de origem latinoamericanas.

No estudo publicado por Delahoy et al (2020), com objetivo de analisar os dados a respeito da hospitalização de 598 gestantes com COVID-19, sintomatologia e desfecho da gestação. Os resultados mostraram quem entre as mulheres hospitalizadas que testaram positivo para SARS-CoV-2 54,5\% estavam assintomáticas na admissão e entre as pacientes sintomáticas (272) 16,2\% (44) foram admitidas em UTI e 8,5\% (23) necessitaram de ventilação mecânica invasiva. O desfecho da gestação é que dos 458 dados disponíveis, 448 tiveram gestação completa com concepto vivo e 10 (2,2\%) resultaram em perda gestacional, sendo essa perda em mulheres sintomáticas e assintomáticas, 4 antes de 20 semanas gestacionais, 5 acima de 20 semanas e 1 com IG desconhecida. Dos 445 conceptos nascidos vivos com idade gestacional conhecida, 87,4\% eram a termo e 12,6\% pré-termo. Entre as mulheres grávidas com dados sobre etnia (577), 42,5\% eram hispânicas e 26,5\% negras. Com relação ao período da gestação das pacientes admitidas que testaram positivo, de 596 mulheres, 14 estavam no primeiro trimestre, 61 no segundo e 521 no terceiro. A razão para hospitalização foi relatada em 324 pacientes, sendo 242 por razões obstétricas, 61 relacionada à COVID-19 e 21 por outras razões. As mulheres hospitalizadas no primeiro e segundo trimestre eram mais frequentemente assintomáticas do que as no terceiro trimestre. Foram relatadas 2 mortes em mulheres sintomáticas. 
Research, Society and Development, v. 10, n. 13, e168101321083, 2021

(CC BY 4.0) | ISSN 2525-3409 | DOI: http://dx.doi.org/10.33448/rsd-v10i13.21083

Tabela 1: Sumarização dos estudos selecionados quanto aos principais aspectos abordados.

\begin{tabular}{lc}
\hline Aspectos observados nos estudos selecionados & Referências \\
\hline & Ellington et al 2020 \\
Grupo étnico & Delahoy et al 2020 \\
& Panagiotakopoulos et al 2020 \\
& Barbero et al. 2020 \\
Lecessidade de internação 2020 \\
Oakes et al 2021 \\
Ellington et al 2020 \\
Panagiotakopoulos et al 2020 \\
Barbero et al. 2020 \\
Li et al. 2020 \\
Chen et al. 2020
\end{tabular}

Idade gestacional no momento da internação

Richtmann et al. 2020

Saccone et al. 2020

Lokken et al 2020

Oakes et al 2021

\begin{tabular}{|c|c|}
\hline Gravidade dos sintomas & $\begin{array}{c}\text { Tekbali et al } 2020 \\
\text { Ayed et al } 2020 \\
\text { Liua et al. } 2020 \\
\text { Caoa et al. } 2020 \\
\text { Knight et al. } 2020 \\
\text { Tug et al. } 2020 \\
\text { Oakes et al } 2021\end{array}$ \\
\hline Necessidade de internação em UTI & $\begin{array}{c}\text { Ellington et al } 2020 \\
\text { Delahoy et al } 2020 \\
\text { Panagiotakopoulos et al } 2020 \\
\text { Zambrano et al } 2020 \\
\text { Remaeus et al. } 2020 \\
\text { Saccone et al. 2020 } \\
\text { Pierce-Williams et al. } 2020 \\
\text { Once et al 2020 } \\
\text { Ferrazzi et al 2020 }\end{array}$ \\
\hline Utilização de ventilação mecânica & $\begin{array}{c}\text { Ellington et al } 2020 \\
\text { Delahoy et al } 2020 \\
\text { Panagiotakopoulos et al } 2020 \\
\text { Zambrano et al } 2020 \\
\text { Remaeus et al. } 2020 \\
\text { Saccone et al. } 2020 \\
\text { Pierce-Williams et al. } 2020 \\
\text { Once et al 2020 } \\
\text { Ferrazzi et al 2020 }\end{array}$ \\
\hline
\end{tabular}

Zambrano et al 2020

Takemoto et al 2020a

Takemoto et al 2020b

Marín et al 2020

Hantoushzadeh et al. 2020

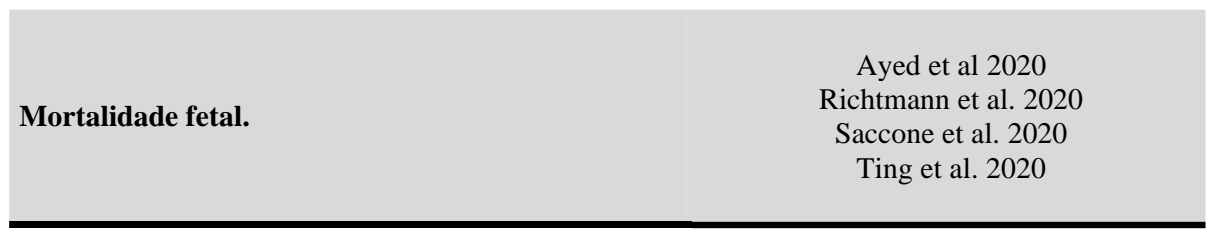

Fonte: Autores (2021).

Neste mesmo sentido de investigação e com resultados similares, Barbero et al (2020), buscaram estudar as características e os resultados de pacientes gestantes hospitalizadas e não hospitalizadas devido a COVID-19. Para isso, foi realizado um estudo de coorte, analisando um total de 91 pacientes confirmadas com o vírus durante a gestação e o puerpério 
(menor que 40 dias desde o parto). Dentre os sintomas manifestados, a maioria apresentou febre, tosse seca, dispneia, calafrios, cefaleia, coriza, perda do paladar e/ou cheiro. Durante o estudo, 40 pacientes $(57,1 \%)$ foram infectadas no terceiro trimestre de gestação (maior que 28 semanas), quatro pacientes precisaram de cuidados na unidade de terapia intensiva (UTI) e quatro pacientes foram diagnosticadas no puerpério (entre 1 e 6 dias), sendo que, destas, uma paciente apresentou piora do quadro respiratório no primeiro dia pós-parto. Além disso, 14 pacientes $(15,4 \%)$ foram hospitalizadas por motivos obstétricos e, destas, cinco foram hospitalizadas por complicações obstétricas antes de desenvolverem os sintomas da COVID-19, sendo: 3 pacientes com rotura prematura de membranas ovulares, 1 gestação gemelar para monitoramento fetal e 1 ameaça de parto prematuro. Corroborando com Delahoy et al (2020) e Barbero et al (2020) em relação à manifestações serem mais presentes em pacientes com IG mais tardias, Lokken e Cols (2020), observaram que 43,5\% estavam no segundo trimestre gestacional e, $50 \%$ estavam no terceiro trimestre, quando da infecção pelo SARS-CoV-2.

Estudo transversal realizado por Panagiotakopoulos e Cols (2020), com objetivo de analisar o motivo de admissão hospitalar em gestantes com COVID-19 e as características da gravidez, como outros fatores de risco. Foram identificadas 105 hospitalizações de gestantes positivas para SARS-CoV-2, sendo que 62 (59\%) por razões obstétricas e 43 (41\%) em consequência da COVID-19 sem razões obstétricas. Das 62 mulheres hospitalizadas por razões obstétricas, 50 estavam assintomáticas para COVID-19. Das 43 hospitalizadas com sintomas de COVID-19, 13 (30\%) precisaram de cuidados em UTI, $6(14 \%)$ precisaram de ventilação mecânica invasiva e 1 faleceu. 44\% das gestantes hospitalizadas por COVID-19 eram obesas, contra $31 \%$ das hospitalizadas por razões obstétricas. O mesmo ocorreu com DM, 26\% das hospitalizadas por COVID19 eram diabéticas contra $8 \%$ das por razões obstétricas. Parto pré termo ocorreu em 15\% das 93 mulheres que deram a luz no período e 3\% eram natimortos. A média de idade foi 30 anos (17-54 anos) e 61,9\% eram latinas. Das 12 mulheres restantes, 1 faleceu e 11 ainda estavam com gestação em andamento.

No estudo de Zambrano e Colaboradores (2020), em que avaliaram 461.825 mulheres com teste positivo para SARSCoV-2 em idade reprodutiva, sendo que 409.462 (88,7\%) dessas estavam sintomáticas, entre as sintomáticas, 23.434 (5,7\%) estavam grávidas. Os dados demonstraram que as gestantes, em relação a não gestante, eram mais susceptíveis à serem admitidas em UTI (10,5 vs 3,9 per 1000), necessitarem de ventilação mecânica invasiva (2,9 vs 1,1 per 1000), receber membrana de oxigenação extracorpórea (ECMO) (0,7 vs 0,3 per 1000) e morrer (1,5 vs 1,2 per 1000). Entre as gestantes, 29,7\% eram latinas/hispânicas. Apesar de mulheres negras fizerem parte apenas de 14,1\% da amostra, elas representaram 36,6\% (176) mortes, sendo 34 (26,5\%) em gestantes. Admissão na UTI foi observada em todos os grupos étnicos, mas foi particularmente notável em asiáticas e nativas das ilhas do pacífico. O risco de receber ventilação mecânica invasiva entre as gestantes foi 3 vezes maior do que não gestantes de 15-34 anos, entre as de 35 a 44 anos esse número foi de 3,6 vezes maior. Além disso, gestantes hispânicas apresentaram 2,4 vezes mais risco de morte. Mulheres grávidas apresentam um risco significativamente maio para formas graves comparadas às não gestantes.

De acordo com Oakes et al (2021), em estudo coorte retrospectivo como objetivo de determinar o risco para a forma severa da COVID-19 entre mulheres grávidas sintomáticas e comparar com as não grávidas usando critérios clínicos padronizados para a forma grave da doença, analisaram 262 mulheres sintomáticas, 22 (8,4\%) eram gestantes e 240 (91,6\%) não gestantes. Entre as gestantes, a maioria eram latinas/hispânicas (36,4\%) e possuíam seguro de saúde público (72,7\%). A média de idade gestacional (IG) foi 32,4 semanas e não houve diferença entre a IG de mulheres com a forma não severa da doença e as com COVID-19 grave. Entre as sintomáticas, 7 (31,8\%) gestantes foram classificadas com a forma severa da COVID-19, necessitando de admissão em UTI.

Segundo os achados de Tekbali e Cols (2020), entre 21.980 admissões, 3064 eram gestantes ou em puerpério. O status de infecção por SARS-CoV-2 para gestantes e puérperas aumentou de $0,14 \%$ na primeira semana para 5,65\% na quarta semana 
Research, Society and Development, v. 10, n. 13, e168101321083, 2021

(CC BY 4.0) | ISSN 2525-3409 | DOI: http://dx.doi.org/10.33448/rsd-v10i13.21083

de pandemia, indicando um risco relativo (RR) de 14,81. Para as não gestantes houve um aumento de $1,21 \%$ na primeira semana para 56,79\% na quarta semana, com RR de 46,99. O diagnóstico foi significativamente maior entre as não gestantes (RR 46,99), mas pode-se relacionar isso a diversos fatores, entre eles o fato de o teste para SARS-CoV-2 não ter sido universal e apenas pacientes sintomáticas realizaram. Pelo fato de gestantes serem mais novas, também são menos susceptíveis a contrair a infecção e desenvolver sintomas, portanto são menos testadas. Também, as admissões para não gestantes estava mais relacionada devido ao parto e questões obstétricas e não necessariamente por sintomas de COVID-19.

No estudo de Takemoto et al (2020), buscaram coletar e analisar dados para ter uma visão geral das mortes maternas relacionadas ao COVID-19 no Brasil, bem como comparar os dados com relatórios mundiais. Para a realização, foi feita uma análise descritiva com base em dados secundários compilados por meio de reportagens na mídia e relatórios epidemiológicos sobre mortes maternas por COVID-19 no sistema de vigilância do Ministério da Saúde do Brasil, relatórios epidemiológicos das Secretarias Estaduais de Saúde e cobertura da mídia. Sendo que, o início dos sintomas foi relatado na gestação em 12 casos $(60,0 \%)$, no pós-parto em 3 casos $(15,0 \%)$, durante a cesárea em 1 caso (5,0\%) e em 4 dados faltantes (20,0\%). Na maioria dos casos, o óbito ocorreu no período pós-parto. Em três casos, o óbito ocorreu na primeira metade da gravidez com 13 (dois) e 22 (um) semanas, respectivamente. Havia 9 gestantes $(45,0 \%)$ sem fator de risco ou sem comorbidade relatada, 9 tinham pelo menos uma comorbidade ou fator de risco e 2 tinham 2 comorbidades ou fatores de risco. A asma foi encontrada em 5 de 11 casos $(45,5 \%)$.

Em outro estudo conduzido por Takemoto e Cols (2020) buscaram descrever os desfechos dos quadros de mulheres gestantes e em puerpério com COVID-19 desde o primeiro caso registrado no Brasil em 26 de fevereiro de 2020 até 18 de junho de 2020, e com isso delimitar possíveis causas para a alta mortalidade de COVID-19 em mulheres grávidas. Este estudo mostrou que de 978 casos positivos, 207 foram admitidos em UTI, sendo 134 recuperados e 73 fatais. $22 \%$ das mulheres que morreram não foram admitidas na UTI e apenas $64 \%$ tinham ventilação invasiva. Nenhuma ventilação foi oferecida em $14,6 \%$ dos casos fatais, enquanto $21,4 \%$ recebeu apensa ventilação não invasiva. Além disso, identificaram diabetes, doença cardiovascular e obesidade como condições significativas associadas à mortalidade na população obstétrica, semelhante à população em geral. Por fim, encontrou 124 óbitos de mulheres grávidas ou puérperas, um número 3,4 vezes maior do que o número total de óbitos maternos relacionados ao COVID - 19 relatados em todo o mundo naquele momento.

Neste mesmo sentido, Ayed et al (2020), buscaram descrever as características clínicas maternas e neonatais e o resultado de gestações com infecção por SARS-CoV-2. Para a realização, foi feito um estudo retrospectivo de base nacional, e análise de prontuários médicos de todas as mulheres grávidas infectadas com SARS-CoV-2 (185 mulheres grávidas infectadas) e seus neonatos. Este estudo mostrou que a maioria (88\%) dessas mulheres apresentava sintomas leves, sendo a febre (58\%) o sintoma mais comum, seguido pela tosse (50,6\%). De 185, 3 (1,6\%) das mulheres grávidas tiveram aborto espontâneo, 2 $(1,1 \%)$ dessas mulheres desenvolveram pneumonia grave e necessitaram de cuidados intensivos.

Corroborando com os achados supracitados, Remaeus et al (2020), estudando 67 gestantes com COVID-19, evidenciram que quatro mulheres $(6 \%)$ foram internadas na unidade de terapia intensiva pós-parto, não houve óbito materno, mas ocorreram dois óbitos perinatais (um neonatal após a ruptura pré-parto das membranas e um natimorto em uma gravidez com restrição de crescimento fetal severa conhecida). Além disso, observaram que das gestantes, $61 \%$ apresentavam sobrepeso ou obesidade, $15 \%$ tinham diabetes e $21 \%$ doença hipertensiva. Setenta por cento das mulheres tiveram parto vaginal, e o parto prematuro ocorreu em $19 \%$ das mulheres, mas apenas duas destas mulheres foram por coronavírus grave. No que tange as consequências da infecção pelo SARS-CoV-2 nas mortes fetais, estudos reportam em uma série de casos de gestantes que evoluíram com óbito fetal que o SARS-Cov-2 foi detectado por RT-PCR em líquido amniótico e placenta. Além disso, foi 
evidenciado corioamnionite aguda na histologia placentária, deposição maciça de fibrina, intervilite/vilite mista e intensa infiltração de neutrófilos e linfócitos (Richtmann et al., 2020; Once et la., 2020).

No estudo de Marian Knight - et al. (2020), buscaram determinar as características e os resultados de mulheres grávidas internadas e infectadas pelo SARS-coV-2 no Reino Unido, desta forma, 427 mulheres grávidas foram investigadas, e os resultados mostraram que a incidência estimada de admissão no hospital com infecção confirmada por SARS-CoV-2 na gravidez foi 4,9 (intervalo de confiança de 95\% 4,5 a 5,4) por 1000 gestantes. E, dentre os principais sintomas, a maioria das pacientes apresentaram febre, tosse e dispneia. Além disso, 104 apresentaram pneumonia no exame radiológico (24\%), 41 (10\%) precisaram de suporte respiratório, 4 mulheres sofreram aborto (1\%) entre 10 e 19 semanas de gestação, e 5 mulheres morreram.

Com relação a idade gestacional influenciar na gravidade da doença, Tug et al. (2020), reportaram em seu estudo avaliando 188 pacientes grávidas que a idade gestacional pode ser um fator que contribui para a gravidade da doença, visto que 13 pacientes $(15,7 \%)$ necessitaram de oxigenoterapia no terceiro trimestre, em relação a 3 pacientes $(8,1 \%)$ no primeiro trimestre e, no segundo trimestre, também foram 3 pacientes $(4,4 \%)$. Ainda em relação a admissão na UTI, a maior taxa ocorreu em pacientes com mais de 20 semanas gestacionais. No intuito de estimar o risco de complicações e necessidade de internação em UTI, Martinez-Portilla et al (2021), avaliaram 5.183 gestantes contaminadas com o vírus. Foi demonstrado que a taxa de pneumonia e admissão na unidade de terapia intensiva (UTI) foi maior em pacientes grávidas em comparação a pacientes não grávidas, todavia a taxa de intubação foi maior em pacientes não grávidas, e a taxa de morte foi igual em ambos os grupos.

\section{Conclusão}

Os resultados encontrados nessa revisão permitiram mapear o perfil epidemiológico das gestantes que desenvolveram COVID-19, concluindo que, as gestantes com os perfis de faixa etária entre 35 e 44 anos, obesidade, asma, doenças cardiovasculares, diabetes, negras, origem latino-americana ou em terceiro trimestre da gestação, apresentaram taxa mais frequente de internações em UTI, ventilação mecânica e critérios de maior gravidade da doença. A gravidez aumenta a morbidade da COVID-19 e esse efeito se torna mais proeminente a medida que a gestação avança. Foi mostrado que com o aumento da prevalência de Sars-Cov-2 nas gestantes, a taxa de óbito materna e neonatal, hospitalização prolongada, admissão na UTI, uso de ventilação mecânica e ECMO subiram. A indicação de cesariana aumentou, devido à emergência por sofrimento fetal ou indicação obstétrica, e as admissões na UTI pós-cesárea aumentaram. Além de que, o número de trabalho de parto pré-termo e subiu por complicações maternas, como também o óbito fetal, por causas como ruptura pré-parto das membranas e aumento da taxa de restrição de crescimento fetal severa conhecida. Evoluções com corioamnionite aguda na histologia placentária de gestações que evoluíram com morte fetal, e eventos tromboembólicos foram detectados na evolução da doença. Quanto aos desfechos neonatais, alguns trabalhos não identificaram nascidos vivos com mãe doente infectados, já outros apresentaram neonatos em proporções pequenas as positividades do PCR para SARS-CoV-2 após o nascimento como também um trabalho mostrou positividade também para a imunoglobulina $\mathrm{M}(\mathrm{IgM})$ e a imunoglobulina $\mathrm{G}$ (IgG) séricas específicas para SARS-CoV. Quanto a testagem para Sars-Cov-2 em líquido amniótico, sangue do cordão umbilical, e amostras de leite materno de seis pacientes, as amostras testaram negativo para o vírus. Trabalhos descartam a possibilidade de transmissão vertical da infecção, porém outros afirmam transmissão ser possível e haver necessidade de mais estudos para confirmar tal afirmação 
Há muitas incertezas quanto aos desdobramentos do vírus na gestação, porém, a atenção obstétrica individualizada e preparada, com bons recursos de pré-natal, monitorização materna e fetal e uma abordagem multiprofissional, se mostraram mais eficazes, visto que os locais com menos recursos em saúde se apresentaram um fator de risco para mortalidade fetal e materna elevada. As informações apresentadas podem sofrer modificações à medida que houver avanços nas descobertas científicas, e pesquisas como as apresentadas poderão servir como base para como protocolos específicos mais eficazes para intervenções ao Sars-Cov2 na gestação, e protocolos para diminuir a transmissão pós-parto para os recém-nascidos.

Como dito, as informações podem sofrer modificações com o surgimento de novas descobertas cientificas. Por isso, sugere-se que os futuros trabalhos tentem compreender melhor as dúvidas que não foram possíveis sanar neste artigo, como os possíveis desdobramentos materno-fetal durante a infecção pelo vírus e a possível transmissão vertical da infecção.

\section{Referências}

Ayed, A., Embaireeg, A., Benawadh, A., Al-Fouzan, W., Hammoud, M., Al-Hathal, M. et al. (2020). Maternal and perinatal characteristics and outcomes of pregnancies complicated with COVID-19 in Kuwait. BMC Pregnancy Childbirth. 20(754):1-9.

Barbero, P., Mugüerza, L., Herraiz, I., García Burguillo, A., San Juan R., Forcén L. et al. (2020). SARS-CoV-2 in pregnancy: characteristics and outcomes of hospitalized and non-hospitalized women due to COVID-19. J Matern Neonatal Med [Internet]. 0(0):1-7 https://doi.org/10.1080/14767058.2020.1793320

Cao, D., Yin, H., Chen, J., Tang, F., Peng, M., Li R. et al. (2020). Clinical analysis of ten pregnant women with COVID-19 in Wuhan, China: A retrospective study. 95:294-00.

Chen, H., Guo, J., Wang, C., Luo, F., Yu, X., Zhang, W. et al. (2020). Clinical characteristics and intrauterine vertical transmission potential of COVID-19 infection in nine pregnant women: a retrospective review of medical records. Lancet. 395:809-15.

Delahoy, M. J., Whitaker, M., Chai, S. J., Daily Kirley, P., Alden, N., Kawasaki B. et al. (2020). Characteristics and Maternal and Birth Outcomes of Hospitalized Pregnant Women with Laboratory-Confirmed COVID-19 - COVID-NET, 13 States, March 1-August 22, 2020. Morbitity Mortal Wkly Rep. 69(38):1347-54.

De Sousa, L. D., Lunardi Filho, W. D., Lunardi, V. L., Santos, S. S. \& Dos Santos, C. P. (2011). The nursing scientific production about the clinic: an integrative review. Rev Esc Enferm, 45 (2), 494-00.

Ellington, S., Strid, P., Tong, V. T., Woodworth, K., Galang, R. R., Zambrano, L. D. et al. (2020). Characteristics of women of reproductive age with laboratory-confirmed SARS-CoV-2 infection by pregnancy status-United States, January 22-June 7, 2020. Morb Mortal Wkly Rep. 69(25):769-75.

Ferrazzi, E., Frigerio, L., Savasi, V., Vergani, P., Prefumo, F., Barresi, S. et al. (2020). Vaginal delivery in SARS-CoV-2-infected pregnant women in Northern Italy: a retrospective analysis. BJOG An Int J Obstet Gynaecol. 127(9):1116-21.

Gabriel, M. A. M., Cuadrado, I., Fernández, B. A., Carrasco, E. G., Díaz, C. A., Martín, I. L. et al. (2020). Multicentre Spanish study found no incidences of viral transmission in infants born to mothers with COVID-19. Acta Paediatr Int J Paediatr. 109(11):2302-8.

Galvão, C. M., Sawada, N. O. \& Trevizan M. A. (2004). Systematic review: a resource that allows the incorporation of evidence into nursing practice. Rev Latino-am Enfermagem, 17 (4), 758-64.

Hantoushzadeh, S., Shamshirsaz, A. A., Aleyasin, A., Seferovic, M. D., Aski, S. K., Arian, S. E. et al. (2020). Maternal death due to COVID-19 Sedigheh. Am J Obstet Gynecol. 223(1):1-16.

Knight, M., Bunch, K., Vousden, N., Morris, E., Simpson, N., Gale, C. et al. (2020). Characteristics and outcomes of pregnant women admitted to hospital with confirmed SARS-CoV-2 infection in UK: National population based cohort study. BMJ. 369.

Li, N., Han, L., Peng, M., Lv, Y., Ouyang, Y., Liu, K. et al. (2020). Maternal and neonatal outcomes of pregnant women with COVID-19 pneumonia: a casecontrol study. Clin Infect Dis. 71(16):2035-41.

Lim, L. M. et al. (2020). Special Report and pregnancy. The American Journal of Obstetrics \& Gynecology. 222(6): 521-31.

Liu, H., Liu, F., Li, J., Zhang, T., \& Wang, D. Clinical and CT imaging features of the COVID-19 pneumonia: Focus on pregnant women and children. $J$ Infect. 2020; 80:7-13.

Lokken, E. M., Walker, C. L., Delaney, S., Kachikis, A., Kretzer, N. M., Erickson, A. et al. (2020). Clinical characteristics of 46 pregnant women with a severe acute respiratory syndrome coronavirus 2 infection in Washington State. Am J Obstet Gynecol. 223(6).

Martinez-Portilla, R. J., Sotiriadis, A., Chatzakis, C., Torres-Torres, J., Espino y Sosa, S., Sandoval-Mandujano, K. et al. (2021). Pregnant women with SARS$\mathrm{CoV}-2$ infection are at higher risk of death and pneumonia: propensity score matched analysis of a nationwide prospective cohort (COV19Mx). Ultrasound Obstet Gynecol. 57(2):224-31. 
Research, Society and Development, v. 10, n. 13, e168101321083, 2021

(CC BY 4.0) | ISSN 2525-3409 | DOI: http://dx.doi.org/10.33448/rsd-v10i13.21083

Oakes, M. C., Kernberg, A. S., Carter, E. B., Foeller, M. E., Palanisamy, A., Raghuraman, N. et al. (2021). Pregnancy as a risk factor for severe coronavirus 2019 (COVID-19) disease using standardized clinical criteria. Am J Obstet Gynecol MFM.;3(3):1-8.

Oncel, M. Y., Alon, I. M., Kanburoglu, M. K., Tayman, C., Coskun, S., Nater, F. et al. (2020). A multicenter study on epidemiological and clinical characteristics of 125 newborns born to women infected with COVID-19 by Turkish Neonatal Society. Eur J Pediatr [Internet]. 180:733-742. https://pubmed.ncbi.nlm.nih.gov/32776309/

Panagiotakopoulos, L., Myers, T. R., Gee, J., Lipkind, H. S., Kharbanda, E. O., Ryan, D. S. et al. (2020). SARS-CoV-2 Infection Among Hospitalized Pregnant Women: Reasons for Admission and Pregnancy Characteristics — Eight U.S. Health Care Centers, March 1-May 30, 2020. Morb Mortal Wkly Rep. 69(38):1355-9.

Pierce-Williams, R. A. M., Burd, J., Felder, L., Khoury, R., Bernstein, P. S., Avila, K. et al. (2020). Clinical course of severe and critical coronavirus disease 2019 in hospitalized pregnancies: a United States cohort study. Am J Obstet Gynecol MFM. 2(3):1-12.

Rajewska, A. et al. (2020). COVID-19 and pregnancy - where are we now? A review. J. Perinat. Med. 48(5): 428-34.

Remaeus, K., Savchenko, J., Brismar Wendel, S., Brusell Gidlöf, S., Graner, S., Jones, E. et al. (2020). Characteristics and short-term obstetric outcomes in a case series of 67 women test-positive for SARS-CoV-2 in Stockholm, Sweden. Acta Obstet Gynecol Scand. 99(12):1626-31.

Richtmann, R., Torloni, M. R., Oyamada Otani, A. R., Levi, J. E., Crema Tobara M, de Almeida Silva C. et al. (2020). Fetal deaths in pregnancies with SARSCoV-2 infection in Brazil: A case series. Case Reports Women's Heal [Internet]. 27:1-5. https://doi.org/10.1016/j.crwh.2020.e00243

Takemoto, M. L. S., Menezes M, de O., Andreucci, C. B., Nakamura-Pereira, M., Amorim, M. M. R., Katz, L. et al. (2020). The tragedy of COVID-19 in Brazil: 124 maternal deaths and counting. Int J Gynecol Obstet. 151(1):154-6.

Takemoto, M. L. S., Menezes, M. O., Andreucci, C. B., Knobel, R., Sousa, L. A. R., Katz, L. et al. (2020). Maternal mortality and COVID-19. J Matern Neonatal Med [Internet]. 0(0):1-7. https://doi.org/10.1080/14767058.2020.1786056

Tekbali, A., Grünebaum, A., Saraya, A., McCullough, L., Bornstein, E., Chervenak, F. A. (2020). Pregnant vs nonpregnant severe acute respiratory syndrome coronavirus 2 and coronavirus disease 2019 hospital admissions: the first 4 weeks in New York. Am J Obstet Gynecol. 223(1):126-7.

Tug, N., Yassa, M., Köle, E., Sakin, Ö., Çakır Köle, M., Karateke, A. et al. (2020). Pregnancy worsens the morbidity of COVID-19 and this effect becomes more prominent as pregnancy advances. J Turkish Soc Obstet Gynecol. 17(3):149-54.

Wenling, Y. et al. (2020). Pregnancy and COVID-19: Management and challenges. Revista do Instituto de Medicina Tropical de Sao Paulo, 62(6): 1-9. Whittemore, R. \& Knafl, K. (2005). The integrative review: updated methodology. J Adv Nurs, 52 (5), 546-53.

Wu, Y. T., Liu, J., Xu, J. J., Chen, Y. F., Yang, W., Chen, Y. F. et al. (2020). Neonatal outcome in 29 pregnant women with COVID-19: A retrospective study in Wuhan, China. PLoS Med [Internet]. 17(7):1-19. http://dx.doi.org/10.1371/journal.pmed.1003195

Wu, Z. \& McGoogan, J. M. (2020). Characteristics of and Important Lessons from the Coronavirus Disease 2019 (COVID-19) Outbreak in China: Summary of a Report of 72314 Cases from the Chinese Center for Disease Control and Prevention. JAMA - Journal of the American Medical Association, 323(13): $1239-42$.

Zambrano, L. D., Ellington, S., Strid, P., Galang, R. R., Oduyebo, T., Tong, V. T. et al. (2020). Update: Characteristics of Symptomatic Women of Reproductive Age with Laboratory-Confirmed SARS-CoV-2 Infection by Pregnancy Status — United States, January 22-October 3, 2020. Morb Mortal Wkly Rep. 69(44):1641-7. 